\title{
Expression of PHA polymerase genes of Pseudomonas putida in Escherichia coli and its effect on PHA formation
}

\section{Journal Article}

Author(s):

Ren, Qun; van Beilen, Jan B.; Sierro, Nicolas; Zinn, Manfred; Kessler, Birgit; Witholt, Bernard

Publication date:

2005-02

Permanent link:

https://doi.org/10.3929/ethz-b-000033180

Rights / license:

In Copyright - Non-Commercial Use Permitted

Originally published in:

Antonie van Leeuwenhoek 87(2), https://doi.org/10.1007/s10482-004-1360-x 


\title{
Expression of PHA polymerase genes of Pseudomonas putida in Escherichia coli and its effect on PHA formation
}

\author{
Qun Ren ${ }^{1, *}$, Jan B. van Beilen ${ }^{2}$, Nicolas Sierro², Manfred Zinn ${ }^{1}$, Birgit Kessler ${ }^{3}$ and \\ Bernard Witholt ${ }^{2}$ \\ ${ }^{1}$ Biocompatible Materials, Swiss Federal Laboratories for Materials Testing and Research (EMPA), \\ Lerchenfeldstrasse 5, 9014 St. Gallen, Switzerland; ${ }^{2}$ Institute of Biotechnology, Swiss Federal Institute of \\ Technology, CH-8093 Zurich, Switzerland; ${ }^{3}$ Präsidialstab, Swiss Federal Institute of Technology, HG F 52.2, \\ CH-8092 Zurich, Switzerland; *Author for correspondence (e-mail: qun.ren-zulian@empa.ch; phone: \\ 41-71-2747688; fax: 41-71-2747788)
}

Received 30 April 2004; accepted in revised form 13 July 2004

Key words: Gene dosage, Gene expression, Heterologous expression, mcl-PHA, PHA monomer composition, Plasmid stability

\begin{abstract}
Poly-3-hydroxyalkanoates (PHAs) are synthesized by many bacteria as intracellular storage material. The final step in PHA biosynthesis is catalyzed by two PHA polymerases (phaC) in Pseudomonas putida. The expression of these two phaC genes (phaCl and phaC2) was studied in Escherichia coli, either under control of the native promoter or under control of an external promoter. It was found that the two phaC genes are not expressed in $E$. coli without an external promoter. During heterologous expression of phaC from Plac on a high copy number plasmid, a rapid reduction of the number of colony forming units was observed, especially for phaC2. It appears that the plasmid instability was partially caused by high-level production of PHA polymerase. Subsequently, tightly regulated phaC2 expression systems on a low copy number vector were applied in E. coli. This resulted in PHA yields of over $20 \%$ of total cell dry weight, which was 2 fold higher than that obtained from the system where phaC2 is present on a high copy number vector. In addition, the PHA monomer composition differed when different gene expression systems or different phaC genes were applied.
\end{abstract}

\section{Introduction}

Medium chain length poly-3-hydroxyalkanoates (mcl-PHAs) accumulated by various bacteria are of increasing industrial interest because of their broad range of potential applications such as biodegradable plastics (Hänggi 1995; Page 1995), crosslinked biodegradable rubbers (de Koning and Witholt 1996), materials in medicine and pharmacy industries (Williams et al. 1999), and sources of chiral monomers (Hrabak 1992). These biodegradable polymers can be produced from renewable substrates and therefore have the potential to replace chemically synthesized polymers, provided that the physiology, genetics, and biochemistry of the PHA-producing organisms are better understood.

Mcl-PHAs were first identified in Pseudomonas putida Gpo1 (earlier known as Pseudomonas oleovorans) grown on $n$-octane and other alkanes (de Smet et al. 1983; van Beilen et al. 2001). It has been reported that the pha locus from $P$. putida GPo1 encodes two PHA polymerases and a depolymerase (Figure 1) (Huisman et al. 1991). The substrate specificities of these enzymes differ slightly (Huisman et al. 1992). The two PHA polymerases are 53\% identical. A homologous operon from $P$. aeruginosa 


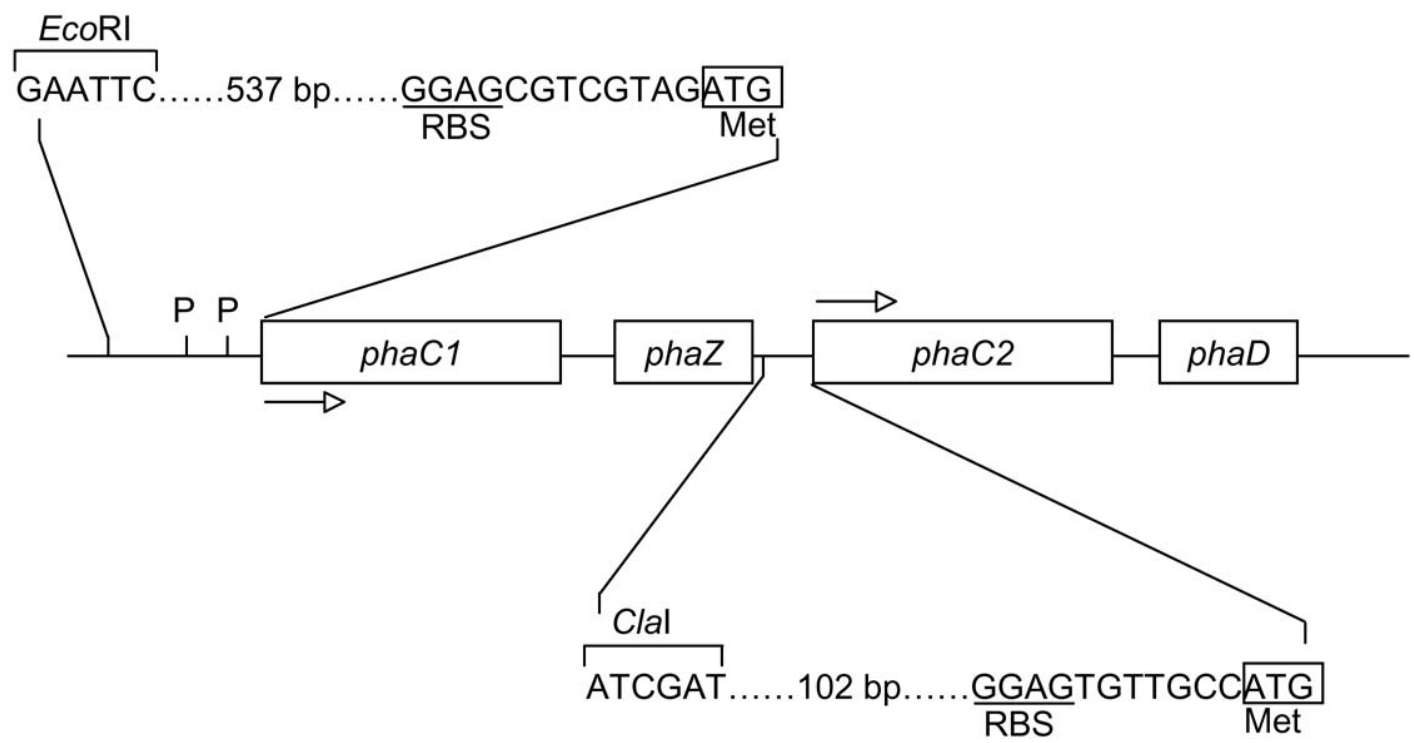

Figure 1. Organization of the pha genes. The scheme (not to scale) summarizes the organisation of the pha locus of $P$. putida Gpo1 containing at least four open reading frames (ORFs) (Huisman et al. 1991). Two polymerases and one depolymerase are encoded by phaC1, phaC2 and phaZ genes, respectively. The phaD gene may encode a granule associated protein (van der Leij and Witholt 1995). Possible promoters are indicated with P. The transcriptional directions are indicated by arrows. The upper and lower parts of the Figure represent expansion of the upstream regions of phaCl and phaC2. The square boxes indicate the start codon. The ribosome binding site (RBS) is underlined. bp, base pair.

has also been identified (Timm and Steinbüchel 1992). The corresponding PHA polymerases from $P$. putida and $P$. aeruginosa show $69-80 \%$ identity at amino acid level (Huisman et al. 1991; Timm and Steinbüchel 1992).

Biosynthesis of these polymers in host organisms that do not naturally produce PHA allows modification of biosynthetic enzymes. This in turn allows increases in the quantity and quality of the products (Kidwell et al. 1995). In addition, introduction of specific genes into an organism having a suitably modified PHA synthetic pathway may allow extension and regulation of the range of compounds that is produced. Escherichia coli is one of these useful hosts. Indeed, many peptides and proteins of pharmaceutical value have been successfully expressed in recombinant E. coli. Mcl-PHA has also been successfully produced in $E$. coli by using $P$. aeruginosa phaC genes (Langenbach et al. 1997; Qi et al. 1997) and $P$. putida GPo1 phaC genes (Ren 1997; Ren et al. 2000a; Ren et al. 2000b) expressed from the Plac promoter. However, there are no reports on the stability and sustainability of heterologous phaC expression systems or high-copy-number plasmids in recombinant E. coli, even though large-scale PHA production requires stable and constant expression of phaC genes.
In this study, we compared expression of the two phaC genes from $P$. putida in E. coli recombinants, and investigated the stability of different phaC expression systems. Our results demonstrated that the two phaC genes cannot be expressed without an external promoter in E. coli. Stable and regulated expression of phaC genes resulted in the formation of about $20 \%$ PHA of total cell dry weight during batch cultivation. Slight differences in the composition of PHA produced with either PhaC1 or PhaC2 were observed. These stable, regulated systems for $p h a C$ gene expressions can serve as a first step toward establishing PHA production in recombinant strains.

\section{Materials and methods}

Strains and plasmids. E. coli fadRfadB strain JMU193 (Rhie and Dennis 1995) and P. putida GPp104 (Huisman et al. 1991) were used throughout. Plasmids pGEc407 (Huisman et al. 1991), pGEc404 (Huisman et al. 1991), pJRD215 (Davison et al. 1987), pGEM-7(+) (Promega), pMMB24 (Bagdasarian et al. 1983), pVLT33/35 (de Lorenzo et al. 1993), pCNB5 (de Lorenzo et al. 1993), pBCKS (Promega), and pUC18/19 (Yanisch-Perron et al. 1985) were used to construct the plasmids listed in Figure 2. Plasmids 
A. Construction of phaCl plasmids

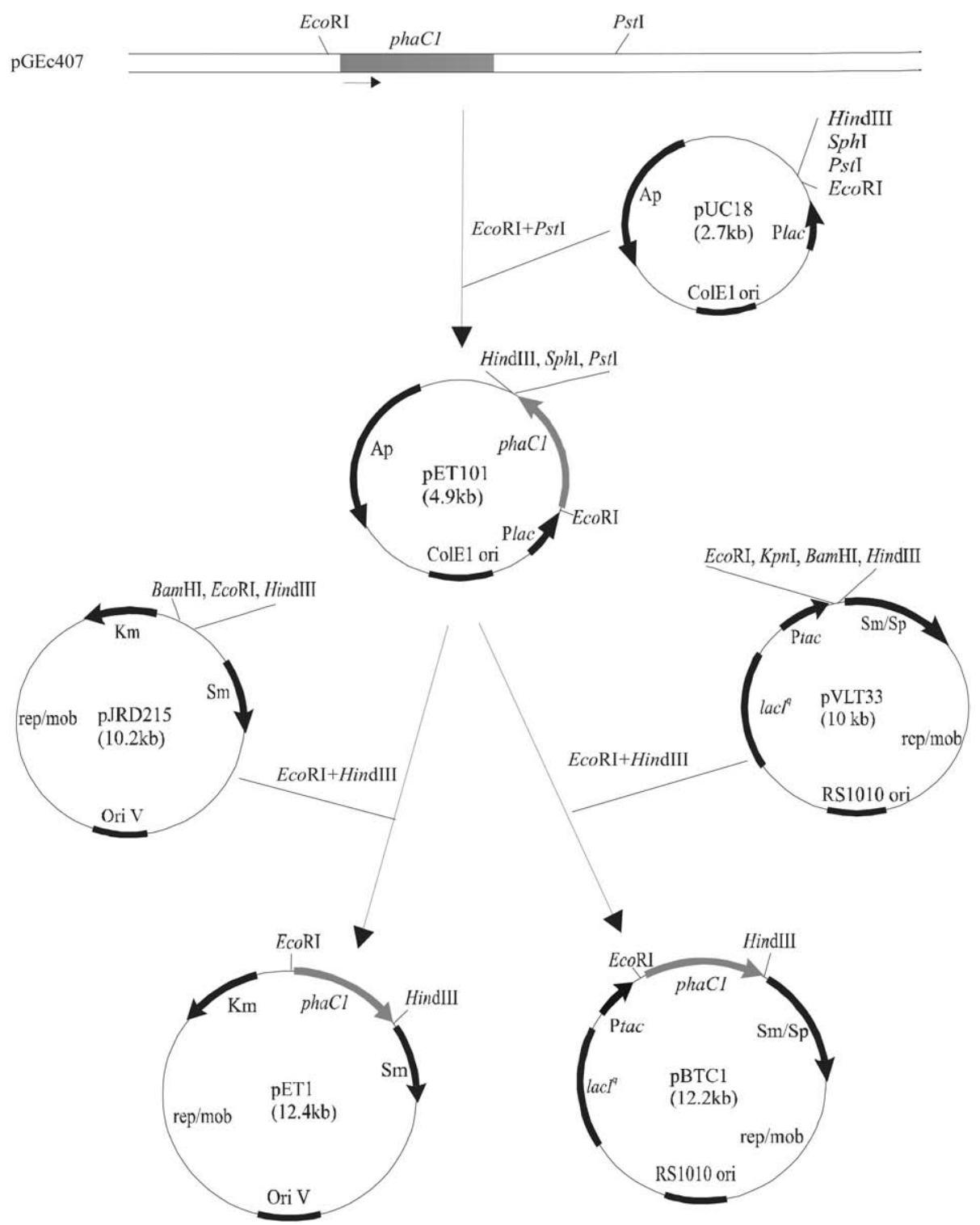

Figure 2. Schematic representation of the strategy to construct plasmids used in this study. Only the relevant cloned gene(s) and restriction site(s) are shown. See Materials \& Methods for further details.

were introduced into JMU193 according to standard procedures (Sambrook et al. 1989).

Recombinant DNA techniques. All general DNA manipulations were performed as previously described (Sambrook et al. 1989). Transformations of $E$. coli competent cells were carried out according to standard procedures (Sambrook et al. 1989).
Construction of pBTC1 and pBTC2 (Figure 2). pET101 was obtained by inserting the EcoRI-PstI phaC1 fragment from pGEc407 in pUC18. pET101 was then cut with EcoRI and HindIII to obtain a 2.2 $\mathrm{kb}$ fragment, which was cloned into the EcoRI and HindIII site of pJRD215, resulting in pET1 (Figure 2 ), or inserted into pVLT33, resulting in pBTC1. pET104 (Ren et al. 2000a) and pET103 contain a 
B. Construction of phaC2 plasmids

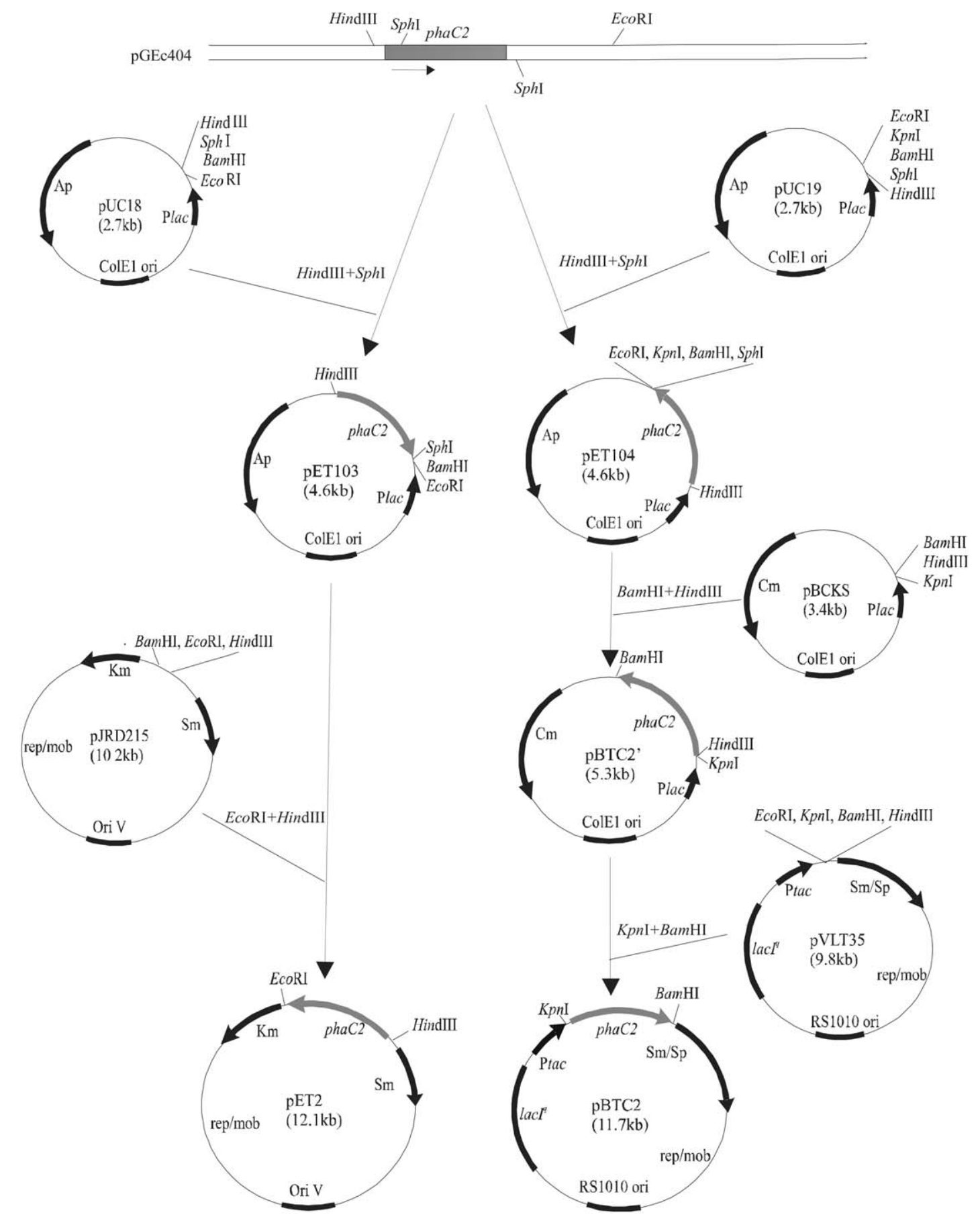

Figure 2. Continued. 


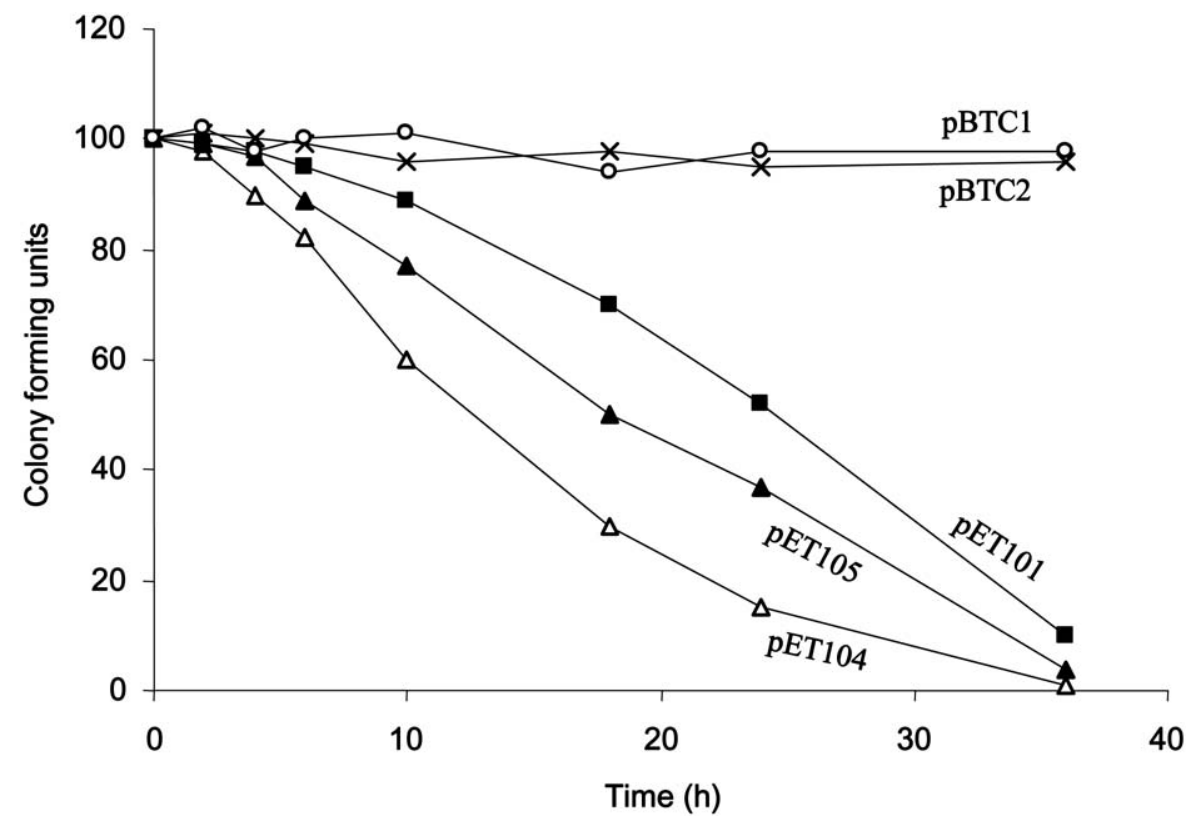

Figure 3. Stability of phaC containing plasmids in E. coli. E. coli recombinants JMU193 carrying pET101, pET104, pET105, pBTC1 and pBTC2 were grown on minimal media containing $2 \mathrm{mM}$ hexadecanoate and $0.2 \%$ yeast extract with relevant antibiotics (ampicillin or kanamycin). Colony forming units were then monitored for $36 \mathrm{~h}$.

HindIII-SphI fragment with phaC2 from pGEc404 in pUC19 and pUC18, respectively. pET105 was derived from pET104 by inserting an EcoRI excised la$c I^{q}$ gene from pCNB5 into pET104. pET2 was obtained by inserting the EcoRI-HindIII fragment with phaC2 from pET103 into pJRD215. pBTC2' (Ren et al. 2000a) was obtained by inserting BamHIHindII fragment of pET104 into pBCKS. pBTC2 (Ren et al. 2000a) contains the HindIII-BamHI fragment with phaC2 from pBTC2' in pVLT35.

Construction of pET2 $\Omega$. pMMB24 was cut with HindIII to obtain the transcriptional terminator $\Omega$, which was then inserted into HindIII digested pET2, resulting in $\mathrm{pET} 2 \Omega$.

Media and culture conditions. E. coli was precultured at $37{ }^{\circ} \mathrm{C}$ in Luria-Bertani (LB) medium (Sambrook et al. 1989), then transferred with 1:100 dilution to 0.1NE2 (Huisman et al. 1989) minimal medium containing $2 \mathrm{mM}$ hexadecanoate and $0.2 \%$ yeast extract as co-carbon source. Hexadecanoate stock solutions were prepared as previously described (Jenkins and Nunn 1987). If necessary, antibiotics were added: kanamycin $(\mathrm{Km}), 50 \mu \mathrm{g} / \mathrm{ml}$, ampicillin (Ap), $150 \mu \mathrm{g} / \mathrm{ml}$, streptomycin (Sp), $50 \mu \mathrm{g} / \mathrm{ml}$, chloramphenicol $(\mathrm{Cm}), 30 \mu \mathrm{g} / \mathrm{ml}$. Cell growth was monitored by measuring optical density at $450 \mathrm{~nm}$ (OD450) (Witholt 1972). In order to induce the Plac or Ptac promoter, isopropyl- $\beta$-D-thiogalactopyranoside (IPTG) was added to indicated concentrations in the early exponential phase (OD450 about 0.2). Samples were taken as described in the results section.

Plasmid stability. To determine the percentage of cells that lost plasmids carrying the ampicillin, streptomycin or kanamycin resistance, cells were serially diluted and plated on LB plates with and without 150 $\mu \mathrm{g} / \mathrm{ml} \mathrm{Ap,} 50 \mu \mathrm{g} / \mathrm{ml} \mathrm{Sp}$ or $50 \mu \mathrm{g} / \mathrm{ml} \mathrm{Km}$. The number of colonies was counted after overnight growth, and compared.

Determination of PHA. To determine the polyester content of bacteria, cells were grown in minimal medium cultures as indicated in the results section and assayed for the presence and composition of PHA by gas chromatography (GC) (Lageveen et al. 1988). All experiments were carried out 2 or 3 times, and averages of these independent experiments are presented.

\section{Results and discussion}

Previously we have reported that deficiency of the fatty acid degradation enzymes FadA or FadB enabled mol-PHA synthesis in E. coli (Ren et al. 
2000a). In this study, the E. coli fadR fadB mutant JMU193 was used. The $f a d R$ gene encodes a protein that exerts negative control over genes necessary for fatty acid oxidation (Black and DiRusso 1994; DiRusso and Nunn 1985; Jenkins and Nunn 1987; Nunn 1986). A mutation in $f a d R$ derepresses transcription of these genes, as a result of which the fad genes are constitutively expressed, rendering $E$. coli capable of growth on mcl fatty acids (Black and DiRusso 1994; DiRusso and Nunn 1985). The $f a d B$ gene encodes four enzyme activities of the fatty acid oxidation path: enoyl-CoA hydratase, 3-hydroxyacylCoA dehydrogenase, $\Delta^{3}$-cis- $\Delta^{2}$-trans-enoyl-CoA isomerase, and 3-hydroxyacyl-CoA epimerase (Black and DiRusso 1994; DiRusso and Nunn 1985; Jenkins and Nunn 1987; Nunn 1986). Mutations in fadB block fatty acid oxidation and result in the accumulation of specific intermediates, which can be channeled to PHA formation (Ren et al. 2000a).

PhaCl and phaC2 expression plasmids. To better understand the consequences of regulated or unregulated $p h a C l$ and $p h a C 2$ expression from their native or heterologous promoters in E. coli, we constructed the plasmids listed in Table 1. Plasmid pET1 contains the native, unmodified phaCl gene plus the upstream region (up to $-555 \mathrm{bp}$ with respect to the ATG start codon) without external promoter (Figure 1). pET2 contains phaC2 plus the upstream region (up to - $120 \mathrm{bp}$ with respect to the ATG start codon) without external promoter (Figure 1). pET101 and pET104 contain phaCl and phaC2, respectively, with upstream regions and the genes are expressed from the Plac promoter (Table 1).

Expression of phaC genes and PHA formation in pha $^{+}$E. coli. E. coli JMU193 was transformed with the plasmids described above. The transformants were cultivated in minimal medium containing $2 \mathrm{mM}$ hexadecanoate and $0.2 \%$ yeast extract as described in 'Material and methods' and samples were analysed for PHA accumulation after $48 \mathrm{~h}$. In E. coli JMU193[pET1], no trace of any of the methyl esters of 3-hydroxyalkanoates $(\leq 0.1 \%$ PHA of cell dry weight) was found (Table 1), whereas in E. coli JMU193 harbouring pET101, a high copy number plasmid through which phaCl is constitutively expressed from the Plac promoter (i. e. the Plac promoter is not controlled by the monocopy lacI product), PHA accumulated to about $26 \%$ of the cellular dry matter (Table 1). Therefore, we conclude that the promoter of phaCl from P. putida GPo1 is not active in E. coli.
In E. coli JMU193 harbouring pET2, about 5\% PHA of total cell dry weight was produced (Table 1), suggesting that a promoter upstream of phaC2 is active in E. coli. To test this possibility, a transcriptional terminator was inserted into the polylinker region -555 bp upstream of phaC2 in pET2, resulting in pET2 $\Omega$ (Table 1). No detectable PHA was found in E. coli JMU193 carrying pET2 $\Omega$. Thus, phaC2 is not expressed in E. coli without an external promoter. The reason why 5\% PHA was found in JMU193 carrying pET2 could be that a putative foreign promoter (such as promoters for antibiotics resistances) upstream of phaC2 is present on pET2, in other words, the expression of phaC2 on pET2 in E. coli is not driven by its native promoter but by a putative foreign promoter upstream of phaC2. Compared with pET2, pET1 did not lead to PHA production in recombinant E. coli, even though both $p h a C l$ from pET1 and phaC2 from pET2 are located on the same plasmid. This could be caused by the reversed orientation of the two genes on the plasmid, resulting in that phaC2 could be expressed by a putative foreign promoter, whereas phaCl could not. When E. coli JMU193 harbouring pET104 constitutive phaC2 expression from the Plac promoter was tested, about 1.2\% PHA was formed (Table 1).

Since each of the phaC genes from P. putida Gpo1 enables PHA synthesis in the PHA negative mutant $P$. putida GPp104, both genes are likely to have a similar function in the formation of PHA (Huisman et al. 1992, Huisman et al. 1991). It has been shown that there is an active promoter upstream of phaCl in $P$. putida (Huisman et al. 1991). A promoter upstream of phaC2 has also been postulated for $P$. aeruginosa PAO1 (Timm and Steinbüchel 1992). Furthermore, expression of pET $2 \Omega$ in P. putida GPp104 resulted in a PHA accumulation of $9.4 \%$ of the total cell dry weight, thereby proving the presence of an active promoter upstream of the phaC2 gene. However, our results demonstrate that these two promoters are not active in $E$. coli. This is different from the well studied PHB synthetic genes, which are constitutively expressed from their own promoter in E. coli (Schubert et al. 1991). Since expression of many Pseudomonas genes is positively regulated, and these genes are not transcribed without the corresponding regulatory protein in E. coli (Deretic et al. 1989), our data indicates that not only the transcription of $\mathrm{phaCl}$ is regulated in P. putida GPo1, as reported previously (Prieto et al. 1999), but also probably that of phaC2. However, we cannot rule out other possibilities for the failure 
Table 1. PHAs produced in E. coli JMU193 carrying different phaC expression plasmids

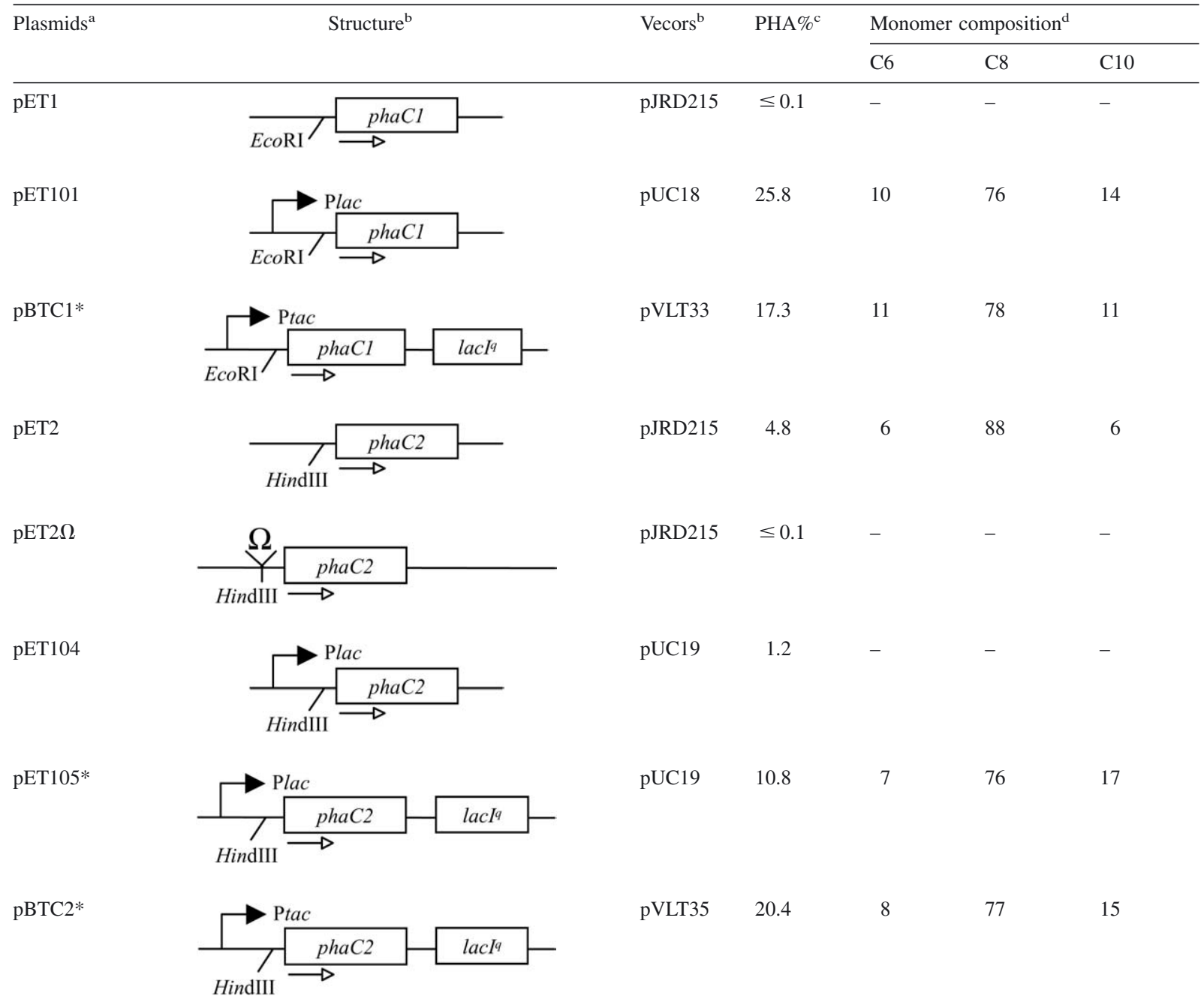

${ }^{\mathrm{a}} E$. coli recombinants were grown on $0.1 \mathrm{NE} 2$ with $2 \mathrm{mM}$ hexadecanoate and $0.2 \%$ yeast extract. Samples were analyzed for PHA after $48 \mathrm{~h}$. *, induced with $100 \mu \mathrm{M}$ or high up to $1 \mathrm{mM}$ IPTG at early exponential phase; ${ }^{\mathrm{b}} \mathrm{pJRD} 215$ (low copy number), pVLT33/35 (low copy number) and pUC18/19 (high copy number) derived plasmids contain the phaCl or phaC2 gene of $P$. putida GPol in orientations indicated by open arrows. The solid arrows represent the direction of the heterologous Plac or Ptac promoter; ${ }^{\mathrm{c}}$ The amount of PHA is given as percentage of cell dry weight (cdw); ${ }^{\mathrm{d}}$ The monomer composition is given as molar percentage. C6, 3-hydroxyhexanoate; C8, 3-hydroxyoctanoate; C10, 3-hydroxydecanoate.

to obtain phaC2 expression in E. coli, such as that the phaC2 promoter is not recognised by the E. coli RNA polymerase.

Plasmid stability in $E$. coli recombinants expressing phaC. One possible reason for the lower amount of PHA associated with the high gene dosage in JMU193[pET104] compared to that in JMU193[pET2] could be the loss of the plasmid. Therefore, we investigated the stability of the constructed plasmids. To determine the percentage of cells that lost the antibiotic resistance, the JMU193 recombinants were grown in batch cultures in minimal media supplied with $2 \mathrm{mM}$ hexadecanoate and $0.2 \%$ yeast extract in the presence of the selective marker (JMU193 carrying pET101 or pET104 with ampicillin, JMU193 carrying pET1 or pET2 with kanamycin), and the antibiotic resistance was monitored for approximately $36 \mathrm{~h}$. Cultures were serially 
diluted and plated on LB plates with and without 150 $\mu \mathrm{g} / \mathrm{ml}$ ampicillin or $50 \mu \mathrm{g} / \mathrm{ml}$ kanamycin. The number of colonies was counted after overnight growth and compared. Plasmids pET1 and pET2 were $100 \%$ stable in JMU193 during the tested time period (data not shown). However, the presence of these plasmids in JMU193 resulted in no or low PHA production (Table 1). Figure 3 shows that in the JMU193[pET104] culture, 60\% of the cells lost the ampicillin resistance within $10 \mathrm{~h}$; while in the JMU193[pET101] culture, 89\% of the cells remained ampicillin resistant after $10 \mathrm{~h}$. The above results reveal that although both phaCl (in pET101) and phaC2 (in pET104) were cloned in the same high copy number plasmid and equipped with the same strong promoter (Table 1), the latter is less stable in E. coli JMU193 (Figure 3). This difference is probably caused by the different upstream regions of phaCl in pET101 and phaC2 in pET104 (Figure 1), or by the nature of the expressed protein.

To test whether uncontrolled expression of phaC2 from pET104 plays a role in the plasmid instability, we constructed pET105 from pET104 (Table 1). pET105 overproduces the LacI repressor protein to control the expression of phaC2. Under induced conditions, about 77\% of the JMU193[pET105] cells remained ampicillin resistant after $10 \mathrm{~h}$ growth in minimal media batch culture with hexadecanoate and yeast extract in the presence of ampicillin (Figure 3). To quantify the PHA content, JMU193[pET105] was cultivated on $0.1 \mathrm{NE} 2$ minimal medium with hexadecanoate and yeast extract as described in 'Materials and Methods'. About 11\% PHA relative to cell dry weight was obtained from JMU193[pET105] after 48 $\mathrm{h}$ (Table 1), whereas less than $0.1 \%$ PHA was found without induction. Therefore, we conclude that uncontrolled overproduction of PHA polymerase in JMU103[pET104] is at least in part responsible for the observed low amount PHA. Regulated expression of phaC2 from pET105 enhanced plasmid stability, which led to enhanced PHA accumulation. The loss of the PHA accumulation phenotype is possibly caused by the segregational instability of pET104 in E. coli JMU193, which could be further increased by the uncontrolled expression of phaC2 from the strong heterologous Plac promoter on a high copy number vector: overproduction of PhaC2 might be toxic to $E$. coli cells. Subsequently, plasmid-free cells grew faster, allowing them to overgrow the recombinant cell population. This hypothesis was confirmed by using pET105. Although pET105 is also not com- pletely stable in E. coli JMU193 (77\% of the cells still contained plasmid after 10 hours), the increased stability compared to pET104 already allowed E. coli JMU193 to synthesize about $11 \%$ PHA upon IPTG induction.

Another reason for the instability of pET101, pET104 and pET105 in JMU193 could be the instability of ampicillin which is degraded in time by $E$. coli cells (Sambrook et al. 1989).

Application of stable plasmids in recombinant $\boldsymbol{E}$. coli. The results described above show that the high copy number plasmids pET101, pET105 and pET104, all with ampicillin markers, were not stable enough for PHA production. This suggests that the combination of controlled phaC expression from a strong heterologous promoter on low copy number plasmid with another antibiotic marker might be effective in stabilizing PHA production in E. coli. Therefore, we constructed pBTC1 and pBTC2 (Figure 2), which are derived from a low copy number vector containing the Ptac promoter, a $\operatorname{lacl}^{q}$ gene, and a streptomycin marker. As shown in Figure 3, both pBTC1 and pBTC2 are much more stable than pET101 and pET104 (or pET105) in E. coli JMU193 when the recombinants were grown in minimal media with hexadecanoate and yeast extract during the tested period. The plasmids were found to be structurally stable as well since the restriction patterns of plasmid DNA isolated during the cultivation were identical to those of the original constructs and they allowed re-transformed JMU193 to accumulate PHA (data not shown).

PHA accumulation using alternative phaC expression systems. E. coli JMU193 containing pBTC2 yielded more PHA than JMU193 containing pET104 or pET105, as shown in Table 1. The PHA content was increased from less than $1 \%$ without induction to about $20 \%$ in response to induction by $100 \mu \mathrm{M}$ IPTG. A similar behaviour was found for JMU193 carrying pBTC1: no detectable PHA was found without induction, while about $17 \%$ PHA was formed when the recombinant was induced with $100 \mu \mathrm{M}$ IPTG (Table 1). Higher IPTG concentrations (up to $1 \mathrm{mM}$ ) had no effect on PHA content or PHA monomer composition in both recombinants.

The PHA content in a host strain depends on the precursor concentration and the polymerase concentration (when it is below a certain level). The precursor concentration is presumably identical in E. coli recombinants JMU193 carrying pET2, pBTC2 and pET105. Since the PHA content (Table 1) differed in 
these E. coli recombinants, these differences must be due to the relative polymerase concentrations. The higher amount of PHA accumulation in JMU193[pET105] (about 11\% of cell dry weight) and JMU193[pBTC2] (about 20\% of cell dry weight) than that found in JMU193[pET2] (about 5\% of cell dry weight) suggests that higher phaC gene dosage can support the accumulation of PHA to a high concentration in recombinant $E$. coli, which has also been observed for poly-3-hydroxybutyrate (PHB) synthesis in E. coli (Lee and Chang 1995, Lee et al. 1994). In other words, relatively large amounts of enzyme seem to be required to direct the carbon flow from 3-hydroxyalkanoates to PHA synthesis among the several competing metabolic pathways such as continued Boxidation (Lee and Chang 1995). The reason why JMU193[pBTC1] produced less PHA (about 17\% of cell dry weight) than JMU193[pET101] (about 26\% of cell dry weight) might be the higher copy number of pET101 than pBTC1, although in both recombinants, phaCl is expressed from the strong promoters Plac or Ptac. However, pET101 is not stable in recombinant E. coli (Figure 3), and thus not suitable for PHA production.

Effect of different phaC genes on the PHA composition. Table 1 shows that when both phaCl and phaC2 genes are located on the same type of plasmid, the PHA polymers formed by $\mathrm{PhaC} 1$ consisted of more C6, similar amounts of $\mathrm{C} 8$ and less $\mathrm{C} 10$ (the molar ratio of C6:C8:C10 is 10:76:14 from pET101, 11:78:11 from pBTC1), compared to polymers produced by $\mathrm{PhaC} 2$ (the molar ratio of $\mathrm{C} 6: \mathrm{C} 8: \mathrm{C} 10$ is 7:76:17 from pET105, 8:77:15 from pBTC2). The PHA monomer composition depends on the precursor concentration, the specificity of the PHA polymerase for each precursor and the polymerase concentration (when it is below a certain level). The precursor concentration and the enzyme specificity profile are presumably identical in E. coli recombinants JMU193 carrying phaC genes. Since the two genes are located on the same type of vector, these PHA composition differences are not likely to be due to a gene dosage or a substrate concentration effect. Instead, these differences suggest that the polymerases have different substrate specificities. Evidently, PhaC1 has higher affinity to 3-hydroxyhexanoyl-CoA and lower affinity to 3-hydroxydecanoyl-CoA than $\mathrm{PhaC} 2$, as was also found for the PHA negative mutant $P$. putida GPp104 carrying each of these two phaC genes (Huisman et al. 1992).

\section{Conclusion}

In this study, we investigated the expression of two phaC genes from P. putida in E. coli. This led to the observation that significant PHA production in $E$. coli requires high phaC1/C2 gene expression. However, the loss of the PHA accumulation phenotype occurred in E. coli recombinants when expression of phaC genes was not tightly controlled, which was similar to poly-3-hydroxybutyrate (PHB) production in $E$. coli (Lee et al. 1994). Apparently, phaC- or phbCABcontaining plasmids are lost when they are not vital to growth and impose detrimental effects on the cells. Therefore, for long term PHA production in E. coli, it is necessary to establish a system which allows stable and high PHA production. In the present report we generated a system where phaC expression was controlled by a strong heterologous promoter from low copy number plasmid and reached stable PHA production in E. coli. However, the PHA levels achieved in $E$. coli recombinants are not yet maximal, compared to that in native and recombinant $P$. putida where PHA is accumulated to $50-70 \%$ of cell dry mass (Huisman et al. 1992). One possibility is that the supply of PHA precursors is rate-limiting in E. coli, thereby limiting the attainable PHA production. Further experiments should be done to address this possibility.

\section{Acknowledgements}

We thank Prof. D. Dennis for providing E. coli strain JMU193. We thank the Swiss Priority Program in Biotechnology for financial support.

\section{References}

Bagdasarian M.M., Amman E., Lurz R., Rückert B. and Bagdasarian M. 1983. Activity of the hybrid trp-lac(tac) promoter of Escherichia coli in Pseudomonas putida. Construction of broadhost-range, controlled-expression vectors. Gene. 931: 273-282.

Black P.N. and DiRusso C.C. 1994. Molecular and biochemical analyses of fatty acid transport, metabolism, and gene regulation in Escherichia coli. Biochimica et Biophysica Acta. 1210: 123145.

Davison J., Heusterspreute M., Chevalier N., Ha-Thi V. and Brunel F. 1987. Vectors with restriction site banks, V. pJRD215, a wide host range cosmid vector with multiple cloning sites. Gene. 51: 275-280. 
de Koning G.J.M. and Witholt B. 1996. A biodegradable rubber from bacteria, poly(hydroxyalkanoate) from Pseudomonas. Mat. Sci. Eng. C. 4: 121-124.

de Lorenzo V., Eltis L., Kessler B. and Timmis K.N. 1993. Analysis of Pseudomonas gene products using lac $I^{q} /$ Ptrp-lac plasmids and transposons that confer conditional phenotypes. Gene. 123: $17-24$.

de Smet M.J., Eggink G., Witholt B., Kingma J. and Wynberg H. 1983. Characterization of intracellular inclusions formed by Pseudomonas oleovorans during growth on octane. J. Bacteriol. 154: 870-878.

Deretic V., Konyecsni W.M., Mohr C.D., Martin D.W. and Hibler N.S. 1989. Common denominators of promoter control in Pseudomonas and other bacteria. Bio/Technology. 7: 12491254.

DiRusso C.C. and Nunn W.D. 1985. Cloning and characterization of a gene $(\mathrm{fadR})$ involved in regulation of fatty acid metabolism in Escherichia coli. J. Bacteriol. 161: 583-588.

Hänggi U.J. 1995. Requirements on bacterial polyesters as future substitute for conventional plastics for consumer goods. FEMS Microbiol. Rev. 16: 213-220.

Hrabak O. 1992. Carbohydrates in industrial synthesis.. In: Clarke M.A. (ed.), Proceedings of the symposium of the division of carbohydrate chemistry of the American Chemical Society. Verlag Dr. Albert Bartens, Berlin, Germany, pp. 18-26.

Huisman G.W., de Leeuw O., Eggink G. and Witholt B. 1989. Synthesis of poly-hydroxyalkanoates is a common feature of fluorescent Pseudomonads. Appl. Environ. Microbiol. 55: 19491954.

Huisman G.W., Wonink E., De Koning G.J.M., Preusting H. and Witholt B. 1992. Synthesis of poly (3-hydroxyalkanoates) by mutant and recombinant Pseudomonas strains. Appl. Microbiol. Biotechnol. 38: 1-5.

Huisman G.W., Wonink E., Meima R., Kazemier B., Terpstra P. and Witholt B. 1991. Metabolism of poly(3-hydroxyalkanoates) (PHAs) by Pseudomonas oleovorans. J. Biol. Che. 266: 21912198.

Jenkins L.J. and Nunn W.D. 1987. Genetic and molecular characterization of the genes involved in short-chain fatty acid degradation in Escherichia coli: the ato system. J. Bacteriol. 169: 4252.

Kidwell J., Valentin H.E. and Dennis D. 1995. Regulated expression of the Alcaligenes eutrophus pha biosynthesis genes in Escherichia coli. Appl. Environ. Microbiol. 61: 1391-1398.

Lageveen R.G., Huisman G.W., Preusting H., Ketelaar P., Eggink G. and Witholt B. 1988. Formation of polyesters by Pseudomonas oleovorans: effect of substrates on formation and composition of poly- $(R)$-3-hydroxyalkanoates and poly- $(R)$-3-hydroxyalkenoates. Appl. Environ. Microbiol. 54: 2924-2932.

Langenbach S., Rehm B.H.A. and Steinbüchel A. 1997. Functional expression of the PHA synthase gene phaCl from Pseudomonas aeruginosa in Escherichia coli results in poly(3-hydroxyalkanoate) synthesis. FEMS Microbiol. Lett. 150: 303-309.

Lee S.Y. and Chang H.N. 1995. Production of poly(3-hydroxybutyric acid) by recombinant Escherichia coli strains: genetic and fermentation studies. Can. J. Microbiol. 41: 207-215.

Lee S.Y., Yim K.S., Chang H.N. and Chang Y.K. 1994. Construction of plasmids, estimation of plasmid stability, and use of stable plasmids for the production of poly(3-Hydroxybutyric acid) by recombinant Escherichia coli. J. Biotechnol. 32: 203211.
Nunn W.D. 1986. Two-carbon compounds and fatty acids as carbon sources.. In: Neidhardt F.C. (ed.), Escherichia coli and Salmonella typhimurium. American Society for Microbiology, Washington, D.C., USA, pp. 285-301.

Page W.J. 1995. Bacterial polyhydroxyalkanoates, natural biodegradable plastics with a great future. Can. J. Microbiol. 41: 1-3.

Prieto M.A., Bühler B., Jung K., Witholt B. and Kessler B. 1999. $\mathrm{PhaF}$, a polyhdroxyalkanoate-granule-associated protein of Pseudomonas oleovorans GPo1 involved in the regulatory expression system for pha genes. J. Bacteriol. 181: 858-868.

Qi Q., Rehm B.H.A. and Steinbüchel A. 1997. Synthesis of poly(3-hydroxyalkanoates) in Escherichia coli expressing the PHA synthase gene phaC2 from Pseudomonas aeruginosa: comparison of PhaC1 and PhaC2. FEMS Microbiol. Lett. 157: 156-162.

Ren Q. 1997. Biosynthesis of medium chain length poly-3hydroxyalkanoates: from Pseudomonas to Escherichia coli. $\mathrm{PhD}$. thesis, ETH Zürich, Switzerland.

Ren Q., Sierro N., Kellerhals M., Kessler B. and Witholt B. 2000a. Properties of engineered poly-3-hydroxyalkanoates (PHAs) produced in Escherichia coli recombinants. Appl. Environ. Microbiol. 66: 1311-1320.

Ren Q., Sierro N., Witholt B. and Kessler B. 2000b. FabG, an NADPH-dependent 3-ketoacyl reductase of Pseudomonas aeruginosa, provides precursors for medium-chain-length poly3-hydroxyalkanoate biosynthesis in Escherichia coli. J. Bacteriol. 182: 2978-2981.

Rhie H.G. and Dennis D. 1995. Role of $\operatorname{fadR}$ and $\operatorname{ato} C(\mathrm{Con}) \mathrm{mu}-$ tations in poly(3-hydroxybutyrate-co-3-hydroxyvalerate) synthesis in recombinant pha ${ }^{+}$Escherichia coli. Appl. Environ. Microbiol. 61: 2487-2492.

Sambrook J., Fritsch E.F. and Maniatis T. 1989. Molecular cloning: a laboratory manual. 2nd edn . Cold Spring Harbor Laboratory Press, New York, USA.

Schubert P., Krueger N. and Steinbuechel A. 1991. Molecular analysis of the Alcaligenes eutrophus poly(3-hydroxybutyrate) biosynthetic operon: Identification of the $\mathrm{N}$ terminus of poly(3hydroxybutyrate) synthase and identification of the promoter. J. Bacteriol. 173: 168-175.

Timm A. and Steinbüchel A. 1992. Cloning and molecular analysis of the poly(3-hydroxyalkanoic acid) gene locus of Pseudomonas aeruginosa PAO1. Eur. J. Biochem. 209: 15-30.

van Beilen J.B., Panke S., Lucchinib S., Franchini A.G., Röthlisberger M. and Witholt B. 2001. Analysis of Pseudomonas putida alkane-degradation gene clusters and flanking insertion sequences: evolution and regulation of the alk genes. Microbiology. 147: 1621-1630.

van der Leij F.R. and Witholt B. 1995. Strategies for the sustainable production of new biodegradable polyesters in plants: a review. Can. J. Microbiol. 41: 222-238.

Williams S.F., Martin D.P., Horowitz D.M. and Peoples O.P. 1999. PHA applications: Addressing the price performance issue I. Tissue engineering. Int. J. Biol. Macromol. 25: 111-121.

Witholt B. 1972. Method for isolating mutants overproducing nicotinamide adenine dinucleotide and its precursors. J. Bacteriol. 109: 350-364.

Yanisch-Perron C., Vieira J. and Messing J. 1985. Improved M13 phage cloning vectors and host strains: nucleotide sequences of the M13mp18 and pUC19 vectors. Genes. 33: 103-119. 